\title{
Is dislocation flow turbulent in deformed crystals?
}

\author{
Woosong Cho:* and Yong S. Chen, Stefanos Papanikolaou, James P. Sethna \\ Laboratory of Atomic and Solid State Physics (LASSP), \\ Clark Hall, Cornell University, Ithaca, New York 14853-2501, USA
}

\begin{abstract}
Intriguing analogies were found between a model of plastic deformation in crystals and turbulence in fluids. A study of this model provides remarkable explanations of known experiments and predicts fractal dislocation pattern formation. Further, the challenges encountered resemble those in turbulence, which is exemplified in a comparison with the Rayleigh-Taylor instability.
\end{abstract}

From horseshoes and knives to bridges and aircrafts, mankind has spent five millennia studying how the structural properties of metals depend not only on their constituents, but also how the atoms are arranged and rearranged as metals are cast, hammered, rolled, and bent into place. A key part of the physics of this plastic distortion is played by the motion of intrinsic line defects called dislocations, and how they move and rearrange to allow the crystal to change shape.

Here, we describe the intriguing analogies we found between our model of plastic deformation in crystals and turbulence in fluids. Studying this model led us to remarkable explanations of existing experiments and let us predict fractal dislocation pattern formation. The challenges we encountered resemble those in turbulence, which we describe here with a comparison to the Rayleigh-Taylor instability.

For brevity, we offer a minimal problem description

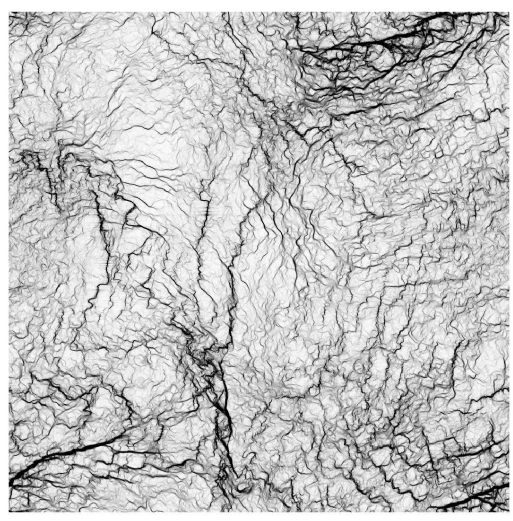

(a) Continuum Dislocation Dynamics

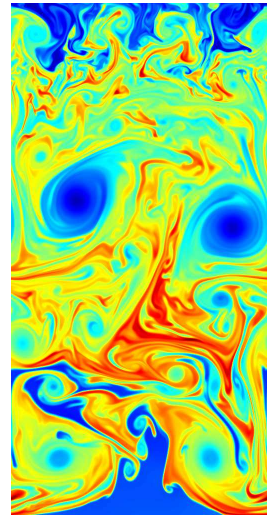

(b) Turbulence
FIG. 1: Comparison of our continuum dislocation dynamics (CDD) with turbulence. (a) Dislocation density profile as it evolves from a smooth random initial condition. The structures form fractal cell wall patterns. Dark regions represent high dislocation density. (b) Rayleigh-Taylor instability at a late time. The fluid (air) with two layers of different densities mix under the effect of gravity. The emerging flows exhibit complex swirling turbulent patterns. The color represents density (red for high, blue for low). that ignores many important features of plastic deformation of crystals, including yield stress, work hardening, dislocation entanglement, and dependence on material properties [1]. We focus on the complex cellular structures that develop in deformed crystals, which appear to be fractal in some experiments [2]. These fractal structures are reproduced by our continuum dislocation dynamics (CDD) [3] theory (see Figure 1a).

Not only do the resulting patterns match the experimental ones, but the theory also has rich dynamics, akin to turbulence. This raises a question: Is the dislocation flow turbulent? Here, we focus on exploring this question by building analogies to an explicit turbulence example: the Rayleigh-Taylor instability. As we describe, our theory displays similar conceptual and computational challenges as does this example, which reassures us that we're on firm ground.

This CDD model [3 5 ] provides a deterministic explanation for the emergence of fractal wall patterns [3, 4] in mesoscale plasticity. The crystal's state is described by the deformation-mediating dislocation density $\varrho_{i j}$ where $i$ denotes the direction of the dislocation lines and $j$ their Burgers vectors [1] - and our dynamical evolution moves this density with a local velocity $V_{\ell}$, yielding a partial differential equation (PDE):

$$
\partial_{t} \varrho_{i j}-\varepsilon_{i m n} \partial_{m}\left(\varepsilon_{n \ell k} V_{\ell} \varrho_{k j}\right)=\nu \partial^{4} \varrho_{i j}
$$

Here $V_{\ell}$ is proportional to the net force on it (overdamped motion), coming from the other dislocations and the external stress. That is,

$$
V_{\ell}=\frac{D}{|\varrho|} \sigma_{m n} \epsilon_{\ell m k} \varrho_{k n}
$$

where $\sigma$ is the local stress tensor, the sum of an external stress $\sigma_{i j}^{\text {ext }}$ and the long-range interactions between dislocations. $\sigma_{i j}^{\text {int }}=\int K_{i j m n}\left(r-r^{\prime}\right) \varrho_{m n}\left(r^{\prime}\right) d r^{\prime}$, with $K_{i j m n}$ the function representing the stress at $r$ generated by $\varrho$ at $r^{\prime}$ [1]. The term proportional to $\nu$ is the regularizing quartic diffusion term for the dislocation density (an artificial viscosity), which we'll focus on here. (In fact, the equation we simulate here is further complicated to constrain the motion of the dislocations to the glide plane while minimizing the elastic energy [3, 4] $]$.) The details of our equations aren't crucial: dislocations move around 
with velocity $\vec{V}$, pushed by external loads and internal stresses to lower their energies. Our equation is nonlin$e a r$, and it's exactly this non-linearity that makes our theory different from more traditional theories of continuum plasticity.

Turbulence is an emergent chaotic flow, typically described by the evolution of the Navier-Stokes equations at high Reynolds numbers:

$$
\begin{aligned}
\rho\left(\partial_{t} \vec{v}+\vec{v} \cdot \nabla \vec{v}\right) & =\mu \nabla^{2} \vec{v}+\vec{f} \\
\partial_{t} \rho+\nabla \cdot(\rho \vec{v}) & =0
\end{aligned}
$$

where $\rho$ is the local density of the fluid with velocity $\vec{v}$ under the application of local external force density $\vec{f}$. The term proportional to $\mu$ is the fluid viscosity, and $\mu$ is inversely proportional to the Reynolds number.

Despite this Navier-Stokes equation's enormous success in describing various experiments, there are many mathematical and numerical open questions associated with its behavior as $\mu \rightarrow 0$. In this regime, complex scale-invariant patterns of eddies and swirls develop in a way that isn't fully understood: turbulence remains one of the classic unsolved problems of science.

How is $\nu$ related to $\mu$ ? Eq. (2) can be written differently by dividing the whole equation by $\rho$, in this equation $\mu / \rho$ (in the incompressible case) is called the kinematic viscosity (usually denoted as $\nu$ ). Our artificial viscosity $\nu$ in Eq. (11) is analogous to this kinematic viscosity. For turbulence, $\mu$ in Eq. (2) is given by nature. In contrast, our $\nu$ in Eq. (1) is added for numerical stability; it smears singular walls to give regularized solutions. This is physically justified because the atomic lattice always provides a cutoff scale. How do we know that this artificial term gives the 'correct' answer (given that there can be many different solutions to the same PDE)? Numerical methods for shock-admitting PDEs are validated by showing that the vanishing grid spacing limit $h \rightarrow 0$ gives the same solution as the $\nu \rightarrow 0$ limit (that is, the viscosity solution). We will argue that both our model of plasticity and the Navier-Stokes equations do not have convergent solutions as $\mu$ or $\nu \rightarrow 0$. We're reassured from the turbulence analogy and are satisfied with extracting physically sensible results from our plasticity theory - viewing it not as the theory of plasticity, but as an acceptable theory.

To solve Eq. (1), we implemented a second order central upwind method [6] especially developed and tested for conservation laws, such as Eqs. (1) and (2). The method uses a generalized approximate Riemann solver which doesn't demand the full knowledge of characteristics [6]. For the simulations of Navier-Stokes dynamics (Eq. (22) ) we use PLUTO [7], a software package built to run hydrodynamics and magnetohydrodynamics simulations, using the Roe approximate Riemann solver.

Accurately capturing singular flows is a challenge in computational fluid dynamics. A classic example of such singularities is the sonic boom that happens when an object passes through a compressible fluid (described by a

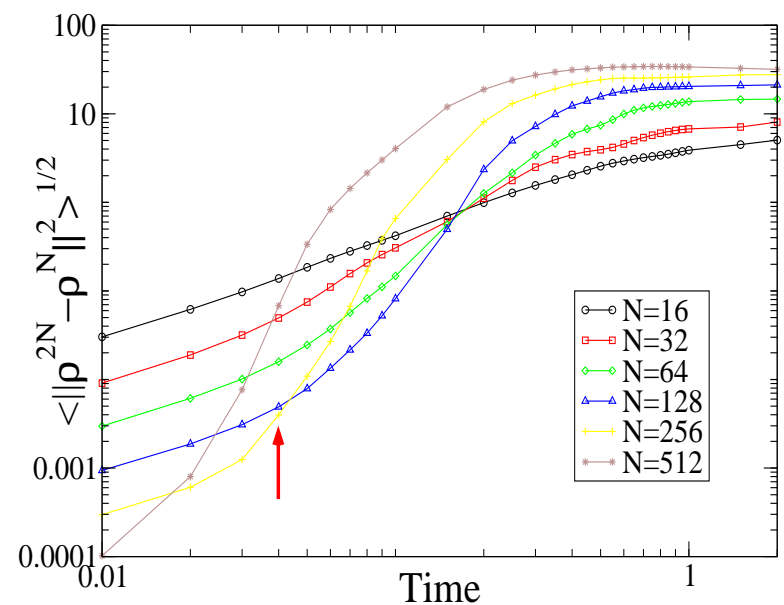

(a) Non-convergence in dislocation dynamics (see Eq. (10)

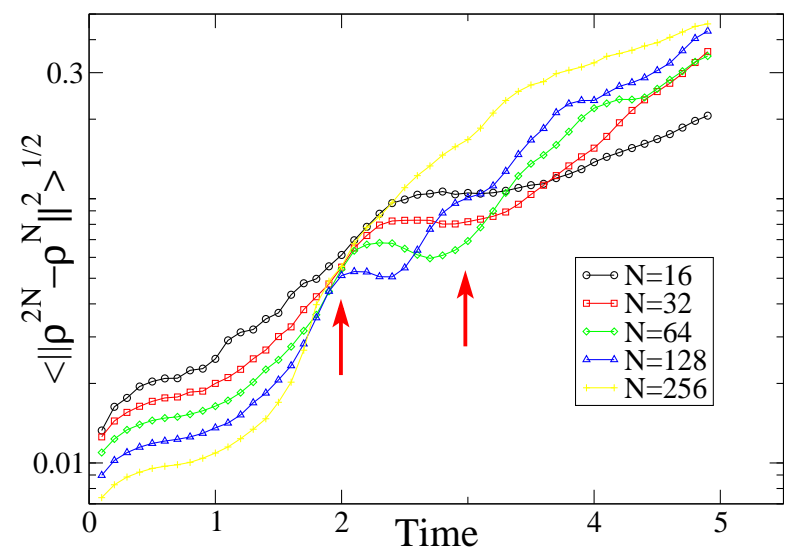

(b) Non-convergence in turbulence

dynamics (Rayleigh-Taylor instability)

FIG. 2: Non-convergence exhibited in both plasticity and turbulence. As time progresses, the curves, which are initially monotonically decreasing, flip order and become non-convergent (where the lines cross each other). Red arrows show where the convergence is lost.

version of Navier-Stokes) faster than its speed of sound. The sonic boom is a sharp jump in density and pressure, which causes the continuum equations to become ill-defined. Our PDEs, depending on gradients of $\varrho$, become ill-defined when $\varrho$ develops an infinite gradient at a dislocation density jump.

The numerical methods we use are designed to appropriately solve the so-called Riemann problem: the evolution of a simple initial condition with a single step in the conserved physical quantities. For hyperbolic conservation laws, exact solutions of the Riemann problem can be obtained by decomposing the step into characteristic waves. However, in most non linear problems, finding exact Riemann solutions involves iterative processes th at are either slow or (practically) impossible, and thus approximate solutions are employed instead. Both methods 
we use are approximate in different ways, but are qualitatively similar.

These sophisticated methods are designed to handle the kind of density jumps seen in sonic booms. In our dislocation dynamics, though, we have a more severe singularity that forms - a sharp wall of dislocations that becomes a $\delta$-function singularity in the dislocation density (as $\nu \rightarrow 0$ ). These $\delta$-shocks are naturally present in crystals - they describe, for example, the grain boundaries found in polycrystalline metals, which (in the continuum limit) form sharp walls of dislocations separating dislocation-free crystallites. Unfortunately, the mathematical and computational understanding of PDEs forming $\delta$-shocks is relatively primitive; there are only a few analytic and numerical studies in one dimension (for an example, see [8]). Currently, to our knowledge, there's no numerical method especially designed for $\delta$-shock solutions. Moreover, in a strict mathematical sense, several properties of Eq. (11) - nonlocality and mixed hyperbolic and parabolic features - haven't been proven to permit a successful application of shock-resolving numerical methods.

In the simulations presented here, we won't add an explicit viscosity (so $\mu=\nu=0$ ); instead, we have an effective numerical dissipation [6] that depends on the grid spacing $h$ as $h^{n}$, where $n$ depends on the numerical method used. (Eq. (2) with $\mu=0$ is the compressible Euler equation. Although we present our simulations as small numerical viscosity limits of Navier-Stokes, they could be viewed as particular approximate solutions of these Euler equations.)

Figure 1 shows typical emerging structures in simulations of both the CDD Eq. (1) and the Navier-Stokes dynamics Eq. (2): both are complex, displaying structures at many different length scales; sharp, irregular walls in the CDD and vortices in Navier-Stokes. Although it might not be surprising to professionals in fluid mechanics that nonlinear PDEs have complex, selfsimilar solutions, it's quite startling to those studying plasticity that their theories can contain such complexity (even though this complexity has been observed in experiments [2]): traditional plasticity simulations do not lead to such structures.

These rich and exotic solutions demand scrutiny. How do we confirm the validity of our solutions? For continuum PDEs solved on a grid, an important problem that needs to be addressed is the effect of the imposed grid. Traditionally, it's expected that as the grid becomes finer, the solution is likely to be closer to the real continuum solution. For differential equations that generate singularities, one cannot expect simple convergence at the singular point! How do we define convergence when singularities are expected? For ordinary density-jump shocks like sonic booms, mathematicians have defined the concept of a weak solution: it's a solution to the integrated version of the original equation, bypassing singular derivatives.
For many problems, researchers have shown that adding an artificial viscosity and taking the limit to zero yields a weak solution to the problem. For some problems, the weak solution is unique, while for others there can be several: different numerical methods or types of regularizing viscosities can yield different dynamics of the singularities. This makes physical sense: if a singular defect (a dislocation or a grain boundary) is defined on an atomic scale, shouldn't the details of how the atoms move (ignored in the continuum theory) be important for the defect's motion? In the particular case of sonic booms, the microscopic physics picks out the viscosity solution (given by an appropriate $\mu \rightarrow 0$ limit), leading mathematicians to largely ignore the question of how micro-scale physics determines the singularities' motion.

However, our problems here are more severe than picking out a particular weak solution. Neither our dislocation dynamics nor the Navier-Stokes equation (with very high Reynolds number) converge in the continuum limit even for gross features, whether we take the grid size to zero in the upwind schemes or we take $\nu \rightarrow 0$ (or $\mu \rightarrow 0$ ) as a mathematical limit.

Figure 2a shows a quantitative measure of the our simulation's convergence as a function of time, as the grid spacing $h=1 / N$ becomes smaller. We measure convergence using the $L_{2}$ norm

$$
\left\|\widehat{\varrho^{2 N}}-\varrho^{N}\right\|_{2} \equiv\left(\int\left\|\widehat{\varrho^{2 N}}-\varrho^{N}\right\|^{2} d x\right)^{1 / 2}
$$

where $\widehat{\varrho^{2 N}}$ has been suitably smeared to the resolution of $\varrho^{N}$. (Normally we'd check the difference between the current solution and the true answer, but here we don't know the true answer.) Here, We study the relaxation of a smooth but randomly chosen initial condition - that is, a perfect single crystal beaten with mesoscale hammers with round heads - as a function of time. We see that for short times these distances converge rapidly to zero, implying convergence of our solution in the $L_{2}$ norm. However, at around $t=0.02$ to 0.2 , the solutions begin to become increasingly different as the grid spacing $h \rightarrow 0$.

This worried us at the beginning because it suggests that the numerical results might be dependent somehow on the artificial finite-difference grid we use to discretize the problem, and therefore might not reflect the correct continuum physical solution. We checked this by adding the aforementioned artificial viscosity $\nu$ in Eq. (1). We found that it converges nicely when $\nu$ is fixed as the grid spacing goes to zero. However, this converged solution is not unique: it keeps changing as $\nu \rightarrow 0$. So, it's our fundamental equation of motion (Eq. (1)) and not our numerical method that fails to have a continuum solution. This would seem even more worrisome: How do we understand a continuum theory whose predictions seem to depend on the smallest studied length scale (the atomic size)? 


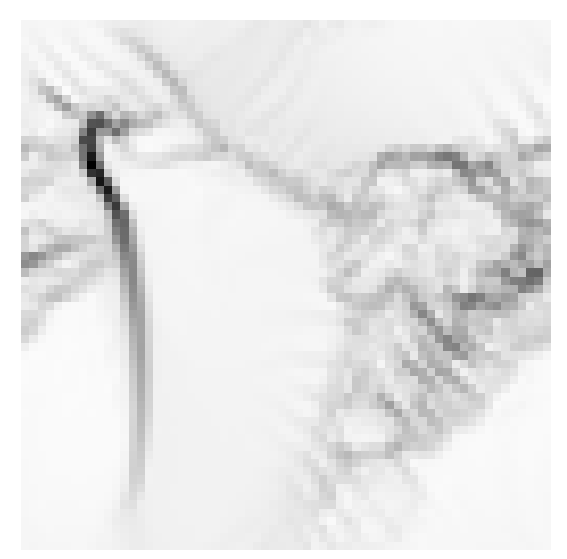

(a) $h=1 / 128$

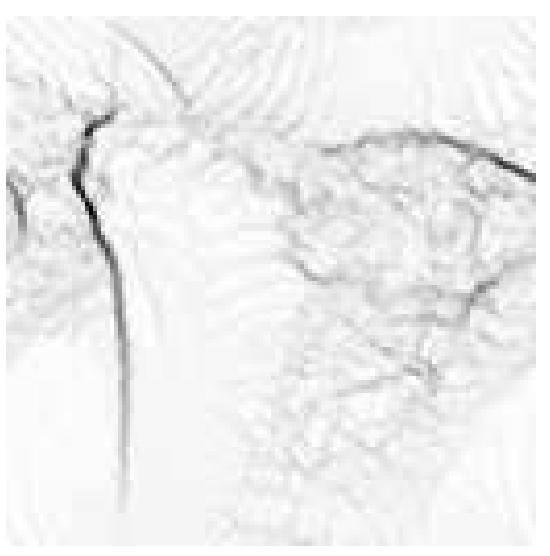

(b) $h=1 / 256$

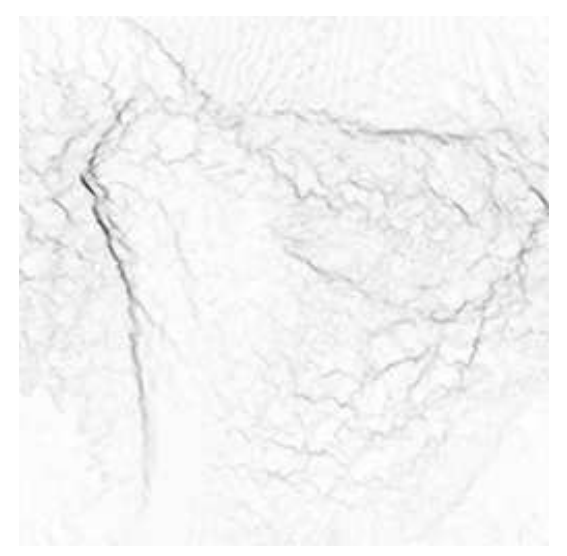

(c) $h=1 / 512$

FIG. 3: Continuum dislocation dynamics. Simulation results at $t=1.0$ of our CDD Eq. (1) at different grid sizes $(h)$, starting from a smooth initial condition. We use periodic boundaries in both horizontal and vertical directions, and all physical quantities are constant along the perpendicular direction.

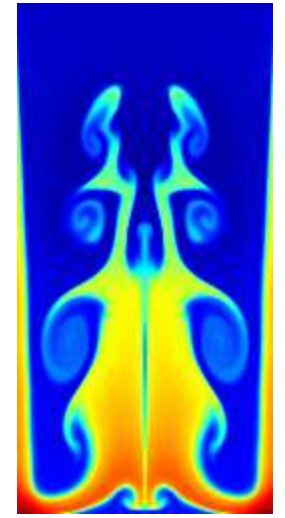

(a) $h=1 / 128$

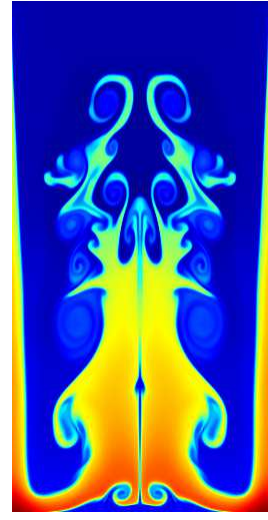

(b) $h=1 / 256$

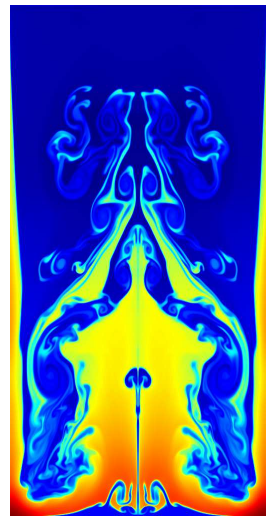

(c) $h=1 / 512$

FIG. 4: The Rayleigh-Taylor instability of the Navier-Stokes equation. The Rayleigh-Taylor instability is a fluid mixing phenomenon that occurs when an interface between two different fluid densities is pulled by gravity. These simulation results here are the Rayleigh-Taylor instability at $t=4.0$, for $\mu \rightarrow 0$, at different grid spacings $(h)$ with periodic boundaries in the horizontal direction and fixed boundaries along the vertical. The initial condition has density interface with a single mode perturbation in the vertical velocity. The system size is $\left(L_{x}, L_{y}\right)=(1.0,2.0)$.

It's here that the analogy to turbulence has been crucial for understanding the physics. It's certainly not obvious that the limit of strong turbulence $\mu \rightarrow 0$ in NavierStokes (Eq. (21)) should converge to a limiting flow. Actually, our short experience suggests that there is no viscosity solution for Eq. (2). Turbulence has a hierarchy of eddies and swirls on all length scales, and as the viscos-

ity decreases (for fixed initial conditions and loading) not only do the small-scale eddies get smaller, but also the position of the large-scale eddies at fixed time change as the viscosity or grid size is reduced.

Figure 2b depicts the convergence behavior of a simulation of the Rayleigh-Taylor instability (as in Fig. 2a). The instability triggers turbulent flow, and like our dislocation simulations, convergence is lost after $t \sim 2.0$ as the grid spacing $h$ gets smaller. Our choice of the Rayleigh-Taylor instability for comparison is motivated by the presence of robust self-similar features (such as the "bubbles" and "spikes" in Fig. [4 [9]), and by the spatiotemporally non-converging features of the initially welldefined interface.

The interface between two fluid densities is analogous to our dislocation cell walls. Even though the RayleighTaylor instability is different from homogeneous turbulence in important ways, we also verified that the latter shows similar spatio-temporal non-convergence but statistical convergence (simulating the Kelvin-Helmholtz instability for compressible flow in 2D). The RayleighTaylor instability provides the best visualization of the analogy between the two phenomena, but this nonconvergence appears to be more general. Fig. 4 shows density profiles at intermediate times for the RayleighTaylor instability. The figure shows the formation of vortices (swirling patterns), and the simulations look significantly different as the grid spacing decreases. Again, this is analogous to the corresponding simulations of our dislocation dynamics shown in Fig. 3, where larger cells continue to distort and shift as the grid spacing decreases.

If the simulations aren't convergent, how can we decide if the theory is physically relevant and can be trusted to interpret experiments? In turbulence, it has long been known that, as vortices develop, self-similar pat- 


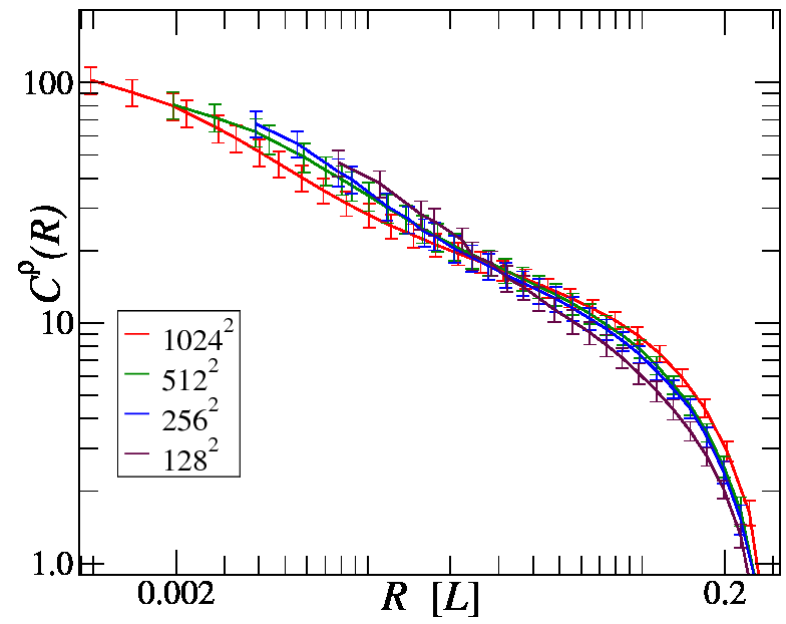

FIG. 5: Statistical properties and convergence Although the continuum dislocation dynamics (CDD) simulations with different grid sizes are non-convergent (Fig. 2a ), the statistical properties are the same. The dislocation density correlation function is plotted here for different simulation sizes at the same time, exhibiting consistent power laws.

terns arise in the flow and exhibit power laws in the energy spectrum and in the velocities' correlation functions [10]. A successful simulation of fully developed turbulence isn't judged by whether the flow duplicates an exact solution of Navier-Stokes! Turbulence simulations study these power laws, comparing them to analytical predictions and experimental measurements.

Our primary theoretical focus in our plasticity study $[3]$ has been to analyze power-law correlation functions for the dislocation density, plastic distortion tensor, and local crystalline orientation. As Fig. 5 shows, like turbulence simulations, these statistical properties seem to converge nicely in the continuum limit.

It's worth noting that, in both cases, non-convergence emerges when small scale features appear on the wall (see Fig. 6) or the interface (see Fig. (7):

In the case of our simulations of plastic flow (Eq.1), starting from smooth initial density profiles, finite time singularities develop in the form of $\delta$-shocks. The existence of finite-time singularities was shown in a $1 \mathrm{D}$ variant of these equations, which is associated with the Burgers equation [11]. Figure [6 shows how this effect occurs by considering the two-norm differences (the integrand in space of the $L_{2}$ norm discussed earlier). At $t=0.04$ (see Fig. 2a) when the $N=512$ curve starts to cross all the other curves, singular features start to appear around a wall (Fig. 6b). Although the boundaries are non-convergent when specific locations and times are considered (Fig. 2a), the statistical properties and associated self-similarity (Fig. 5) are convergent.

In the case of Navier-Stokes simulations (see Fig. 17), the existence of finite-time singularities is a topic of active research: even though local-in-time analytic solutions are easily shown to exist, global-in-time analytic solutions can be proven to exist only for special cases, such as in the 2D incompressible genuine Euler equation [12]. In $3 \mathrm{D}$, the mechanism of vortex stretching is conjectured to lead to finite-time singularities [13], even though there are still crucial open questions. Despite its complexity, turbulence can be concretely studied in special cases. For our example of the Rayleigh-Taylor instability, the twofluid interface gets distorted and "bubbles" form (Fig. (4); over time, the bubbles exhibit emergent self-similar characteristics [9], showing statistical convergence. However, there is no spatio-temporal convergence, because the interface develops complex, turbulent features as the grid becomes finer (see Figs. 2b and (7).

Sometimes science seems to be fragmented, with independent fields whose vocabularies, toolkits, and even philosophies almost completely separate. But many valuable insights and advances arise when ideas from one field are linked to another. Computational science is providing a new source of these links, by tying together fields that can fruitfully share numerical methods.

Our use of well-established numerical methods from the fluids community made it both natural and easy to utilize their analytical methods for judging the validity of our simulations and interpreting their results.

We thank Alexander Vladimirsky, Randall LeVeque, Chi-Wang Shu, and Eric Siggia for helpful and inspiring discussions on numerical methods and turbulence. The US Department of Energy/Basic Energy Sciences grant no. DE-FG02-07ER46393 supported our work, and the US National Center for Supercomputing Applications partially provided computational resources on the Lincoln and Abe clusters through grant no. MSS090037.

* Electronic address: wc274@cornell.edu

$\dagger$ Electronic address: sethna@lassp.cornell.edu

[1] T. Mura, Micromechanics of defects in solids (Springer, 1987), 2nd ed.

[2] P. Hähner, K. Bay, and M. Zaiser, Phys. Rev. Lett. 81, 2470 (1998).

[3] Y. S. Chen, W. Choi, S. Papanikolaou, and J. P. Sethna, Phys. Rev. Lett. 105, 105501 (2010).

[4] S. Limkumnerd and J. P. Sethna, Phys. Rev. Lett. 96, 095503 (2006).

[5] A. Acharya, J. Mech. Phys. Solids 49, 761 (2001).

[6] A. Kurganov, S. Noelle, and G. Petrova, SIAM J. Sci. Comput. 23, 707 (2002).

[7] A. Mignone, G. Bodo, S. Massaglia, T. Matsakos, O. Tesileanu, C. Zanni, and A. Ferrari, Astrophys. J. Suppl. S. 170, 228 (2007).

[8] D. C. Tan, T. Zhang, T. Chang, and Y. X. Zheng, J. Differ. Equations 112, 1 (1994).

[9] J. Shi, Y.-T. Zhang, and C.-W. Shu, J. Comput. Phys. 


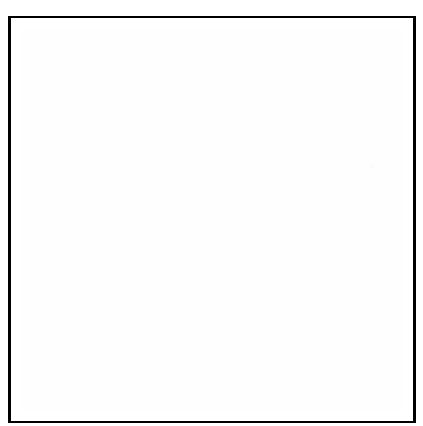

(a) $h=1 / 256$

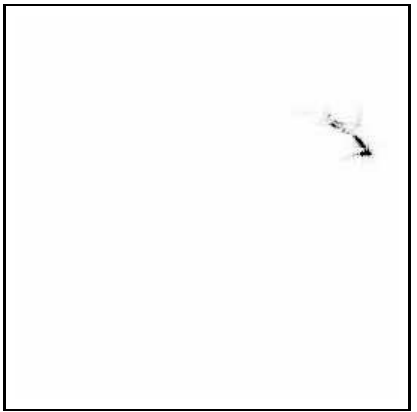

(b) $h=1 / 512$

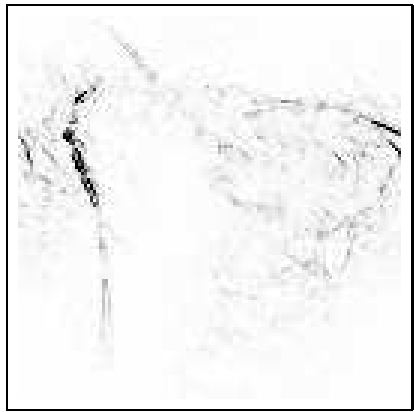

(c) $h=1 / 256$

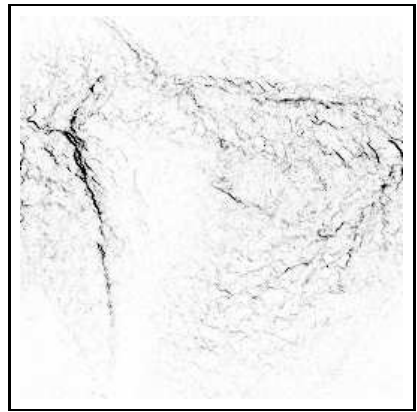

(d) $h=1 / 512$

$$
t=0.04
$$

FIG. 6: Non-convergence and singularity for CDD For (a) and (b) $t=0.04$; for (c) and (d), $t=1.00$. The two-norm difference between $h$ and $h / 2$ are plotted. At short time, $t=0.04$, the differences are small: (a) is empty and (b) nearly so. At later times, $t=1.00$, the two-norm difference becomes significant esepcially where the walls are forming (see Fig. 3 for wall locations).

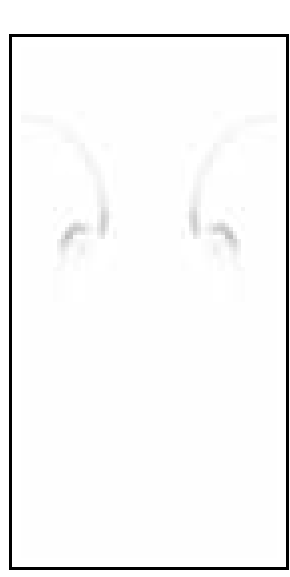

(a) $h=1 / 64$

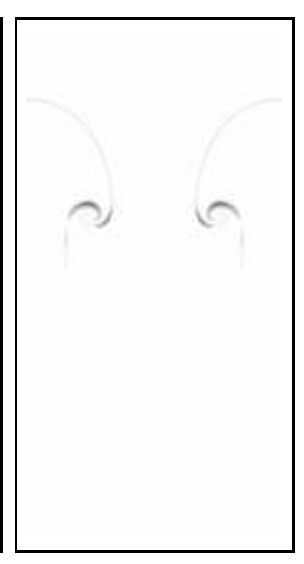

(b) $h=1 / 128$ $t=2.0$

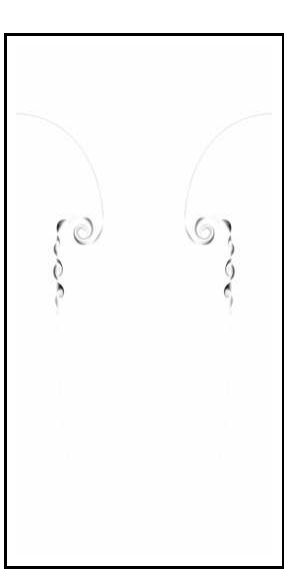

(c) $h=1 / 256$

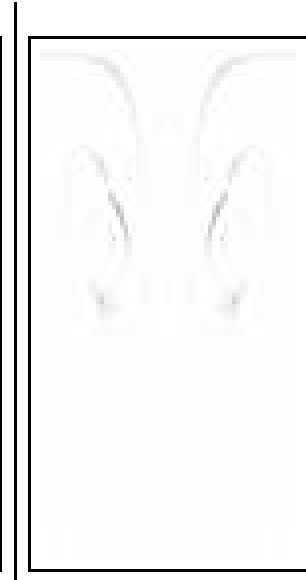

(d) $h=1 / 64$

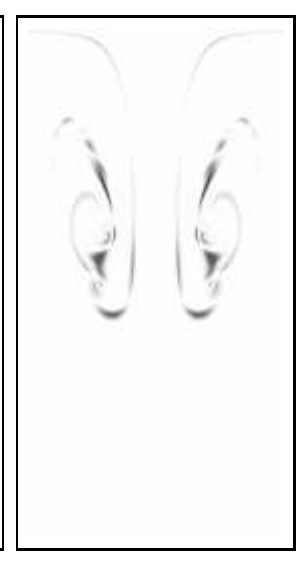

(e) $h=1 / 128$ $t=3.0$

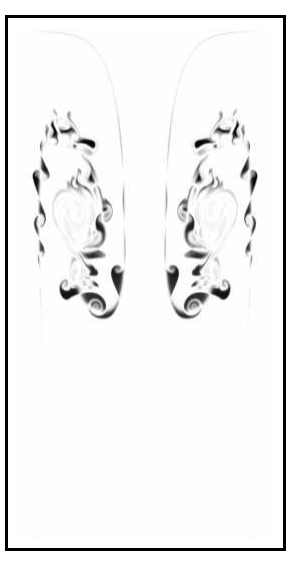

(f) $h=1 / 256$

FIG. 7: Non-convergence and vortices for Navier-Stokes For (a) (b), and (c) $t=2.0$; for (d), (e) and (f) $t=3.0$, corresponding to the two red arrows in Figure 2b The two-norm difference between $h$ and $h / 2$ is plotted. At $t=2.0$, small structures start to become strong, as in (c), and the same is true at $t=3.0$ for $h=1 / 128$, as in (e).

186, 690 (2003).

[10] U. Frisch, Turbulence : the legacy of A.N. Kolmogorov (Cambridge University Press, 1995).

[11] S. Limkumnerd and J. P. Sethna, J. Mech. Phys. Solids 56, 1450 (2008).
[12] C. Bardos and D. Lannes, e-print arXiv:1005.5329 (2010).

[13] A. Pumir and E. D. Siggia, Phys. Rev. Lett. 68, 1511 (1992). 